\title{
Phase transition in tensionless surfaces
}

\author{
J.J. Ruiz-Lorenzo ${ }^{\mathrm{a}, \mathrm{b}, *}$, R. Cuerno ${ }^{\mathrm{c}}$, E. Moro ${ }^{\mathrm{c}}$, A. Sánchez ${ }^{\mathrm{b}, \mathrm{c}}$ \\ a Departamento de Física, Facultad de Ciencias, Universidad de Extremadura, 06071 Badajoz, Spain \\ ${ }^{\mathrm{b}}$ Instituto de Biocomputación y Física de Sistemas Complejos (INBIFI), Facultad de Ciencias, Univesidad de Zaragoza, 50009 Zaragoza, Spain \\ ${ }^{\mathrm{c}}$ Departamento de Matemáticas and Grupo Interdisciplinar de Sistemas Complejos (GISC), Universidad Carlos III de Madrid, E-28911, Leganés, Spain
}

\begin{abstract}
We study the critical behavior of the Laplacian roughening model, which describes the growth of tensionless surfaces. This type of growth phenomena is very important, for instance, in biological membranes and in molecular beam epitaxy. We summarize previous analytical and numerical results and point out their contradictions and differences, thus making clear the context of our work. Our contribution, achieved through large scale numerical simulations, is the confirmation that the model exhibits a single continuous phase transition: the transition takes place between a continuum massless (i.e., with infinite correlation length) bilaplacian behavior and a massive propagator (finite correlation length).
\end{abstract}

Keywords: Statistical Physics; Surfaces

\section{Introduction}

Tensionless surfaces are of great importance in different fields and applications, ranging from biology, where lipid membranes are tensionless for practical purposes [1], to nanotechnology, where molecular beam epitaxy and related techniques used to grow devices are carried out in conditions for which surface tension is negligible [2]. Therefore, from the viewpoint of these and other applications, understanding the different regimes in which tensionless surfaces can grow is a very relevant issue. In this context, in this paper we study the critical behavior of a model which describes tensionless surfaces. In spite of its simplicity, the model has been the subject of a number of studies [3-8], which have led to disparate and often contradictory predictions of its behavior. Our aim in this work is to carry out large-scale numerical simulations

* Corresponding author. Departamento de Física, Facultad de Ciencias, Universidad de Extremadura, 06071 Badajoz, Spain. Tel.: +34 924 289300; fax: +34924289651.

E-mail address: ruiz@unex.es (J.J. Ruiz-Lorenzo). that can clarify the actual scenario in which the transition (or transitions) takes place. Indeed, one of the most intriguing previous results is the conjecture [3] that the model undergoes two different phase transitions between the so-called liquid, hexatic, and solid phases (names borrowed from studies of two-dimensional melting, but standard in this context). As we will see below, our numerical results do not support this picture and allow us to claim that there is no hexatic phase in the model. Furthermore, we find that the transition is continuous, contrary to earlier simulations [5] which reported a firstorder transition.

In order to present clearly our conclusions, the outline of the paper is as follows. In the next two subsections we motivate the definition of the model and recall briefly examples of its applications. We then (Section 2) establish our notation and define the observables we study. Section 3 is devoted to summarizing all available analytical and numerical results to date. Section 4 reports the outcome of our numerical simulation program. Finally, we recapitulate on our work and its relation to previous ones in the conclusions, in Section 5. 


\subsection{Tensionless surfaces}

Generally speaking (see, e.g., [2] for a detailed introduction to the subject), the free energy of a surface $(F=\mu N-p V+\sigma A)$ can be written as follows:

$F=\left(\frac{\mu}{v}-p\right) \iint \mathrm{d} x \mathrm{~d} y h(x, y)+\iint \mathrm{d} x \mathrm{~d} y \phi\left(h_{x}, h_{y}\right)$,

where $\mu$ is the chemical potential, $v=V / N$ is the specific volume, $h(x, y)$ is the height of the surface above point $(x, y)$, $p$ is the pressure, and $\phi\left(h_{x}, h_{y}\right)=\sigma\left(h_{x}, h_{y}\right) \sqrt{ } 1+h_{x}^{2}+h_{y}^{2}$, with $\sigma$ being the surface tension and $A$ being the area. Working at constant pressure, we can study the in influence of either the chemical potential or the surface tension in the way the surface grows.

First, the main effect of the chemical potential is to induce diffusion on the surface from regions with higher chemical potential to regions with lower chemical potential. A conserved mass current (the number of particles is constant) is generated that is restricted to the surface, reads

$j=-D_{\mathrm{s}} \nabla \mu$,

and drives dynamics for the surface height, in the form of a continuity equation

$\frac{\partial h}{\partial t}=-\nabla \cdot j$

Combining both expressions, and having in mind that, to linear order, the chemical potential (that is proportional to the mean surface curvature) is related to the surface height by $\mu \propto-\nabla^{2} h$, we arrive at

$\frac{\partial h}{\partial t}=D_{\mathrm{s}} \nabla^{2} \mu=-\kappa\left(\nabla^{2}\right)^{2} h, \quad$ with $\kappa>0$.

Second, let us consider the other ingredient, surface tension. Assuming that the chemical potential of the vapor is uniform, $\mu_{0}$, there will be evaporation in places where $\mu>\mu_{0}$. We can thus write the variation of the height with time as a linear function of the chemical potential difference:

$\frac{\partial h}{\partial t} \propto-\left(\mu-\mu_{0}\right)$

and so

$\frac{\partial h}{\partial t}=v \nabla^{2} h, \quad$ with $v>0$.

If we now include all the above factors in our description, i.e., we take into account evaporation, surface diffusion and thermal fluctuations, we can write the following Langevin equation for the surface height

$\frac{\partial h}{\partial t}=v \nabla^{2} h-\kappa\left(\nabla^{2}\right)^{2} h+\eta(\mathbf{r}, t)$ where $\eta(\mathbf{r}, t)$ is a Gaussian white noise. This is the generalized, continuum Laplacian roughening model. ${ }^{1}$ From this general expression, by letting $\nu=0$, we are left with a model for continuum tensionless surfaces, henceforth referred to as the bilaplacian model.

\subsection{Examples of tensionless surfaces}

One example of a tensionless surface is provided by a lipid membrane [1]. The fluid of lipids which conforms the membrane is above its melting point and hence lipids can move inside the membrane. Recently Gov and Safran [9] have proposed the following equation to model the action of the elastic cytoskeleton on the fluid membrane:

$\kappa\left(\nabla^{2}\right)^{2} \chi(\mathbf{r})+V(\mathbf{r}) \chi(\mathbf{r})=0$

where $\chi(\mathbf{r})=\langle h(\mathbf{r})\rangle$ (i.e., we are in a mean field approximation), and $V(\mathbf{r})$ represents the pinning effect of the cytoskeleton on the surface. Eq. (2) is the mean field analog of Eq. (1) for $v=0$, in the presence of an external potential.

A second instance of tensionless surfaces arises in the growth of thin films by molecular beam epitaxy (MBE) $[2,6]$. MBE is a process of high technological interest, in which atoms or molecules are deposited on the surface of the growing sample in conditions such that evaporation is largely suppressed. This suppression leads in turn, following the reasoning of the above subsection, to the continuum bilaplacian model [Eq. (1) with $v=0$ ], referred to in this context as the linear MBE equation.

Finally, the third example is two-dimensional melting. Nelson [3] proposed the Laplacian roughening model in order to describe this phenomenon. We specify details of this model below. We refer the reader to the original work for details on how two-dimensional melting occurs within such approach.

\section{Model, observables, and their meaning}

The Hamiltonian of the generalized Laplacian roughening model is

$\mathcal{H}=\sum_{\mathbf{r}}\left(v\left[\nabla_{\mathrm{d}} h(\mathbf{r})\right]^{2}+\kappa\left[\nabla_{\mathrm{d}}^{2} h(\mathbf{r})\right]^{2}\right)$

where $\mathbf{r}$ is denotes position on a (square) two-dimensional lattice, $\nabla_{\mathrm{d}}$ is the corresponding discrete gradient and, importantly, $h \in \mathbb{Z}$. The original discrete Laplacian roughening model [3] is obtained by letting $\nu=0$.

In order to characterize the model and its possible phases, it is interesting to define two different correlation functions

\footnotetext{
${ }^{1}$ We can take into account an external flux of particles, $F$, by performing the transformation $h \rightarrow h+F t$.
} 
(or propagators). The first one, the usual height difference correlation function, is defined as

$C(\mathbf{r}) \equiv\left\langle(h(\mathbf{r})-h(0))^{2}\right\rangle$,

where the brackets denote thermal average, whereas the slope-slope correlation function is given by

$C_{\mathrm{d}}(s) \equiv\left\langle\left(\nabla_{\mathrm{d}} h(\mathbf{r}+s)-\nabla_{\mathrm{d}} h(\mathbf{r})\right)^{2}\right\rangle$.

In the continuum model (i.e., continuous heights, $h \in \mathbb{R}$ ) the previous two propagators, as we will refer to them in the following, behave in momentum space as $C(q) \propto 1 / q^{4}$ and $C_{\mathrm{d}}(q) \propto 1 / q^{2}$, respectively, as can be easily seen by solving the linear Eq. (1) in Fourier space. We can define the surface tension as the coefficient of the quadratic term (in the momentum variable) in $1 / C(q)$. If a quadratic term is observed in numerical simulations, it will indicate that the surface has developed an effective surface tension. On the other hand, if no quadratic term is present, we will use the term tensionless to describe the corresponding situation: the surface tension is absent. Finally, as a standard magnitude to look for phase transitions, we define the specific heat, $c$, as

$c=\frac{1}{V}\left(\left\langle\mathcal{H}^{2}\right\rangle-\langle\mathcal{H}\rangle^{2}\right)$,

where $V=L^{2}$ is the volume of the two-dimensional lattice.

\section{The Laplacian roughening model: previous results}

Before reporting our results, we have to summarize previous analytical and numerical results in order to place our findings in their proper context. Therefore, we will now describe, briefly, results obtained using three different analytical methods: mapping to a two-dimensional vector Coulomb gas [3], mean field [7], and renormalization group
(RG) (dynamics) [8]. Subsequently, we will recall the available numerical results $[4,5]$.

Beginning with the analytical approaches, as we will see below, the predictions of the aforementioned techniques lead to largely discrepant results. Without going into much technical detail, we can sum up the main conclusions as follows:

Nelson Scenario [3]. As mentioned above, the application of interest in this case was two-dimensional melting. In any event, by using the Poisson summation formula the Laplacian roughening model [Eq. (3) with $\nu=0$ ] can be written as a vector Coulomb gas, in which the roles of high and low temperatures are reversed. Taking into account the most relevant terms in the modified Coulomb gas, a sequence of two Kosterlitz-Thouless (KT) transitions was found separating three phases. In terms of the propagators defined above, those phases were characterized by:

(1) $T>T_{\mathrm{c} 1}: C(r) \propto r^{2} \log r, C_{\mathrm{d}}(r) \propto \log r$ (solid phase).

(2) $T_{\mathrm{c} 1}>T>T_{\mathrm{c} 2}: C(r) \propto \log r, C_{\mathrm{d}}(r) \propto 1$ (hexatic phase).

(3) $T<T_{\mathrm{c} 2}: C(r) \propto 1, C_{\mathrm{d}}(r) \propto 1$ (isotropic liquid phase).

In parenthesis, we have collected the physical meaning of the different phases in the melting context. See [3] for further details.

Mean Field Scenario [7]. In this approach, the starting point is the Bogoliubov-Feynman inequality for the free energy:

$$
\begin{aligned}
& \mathcal{F} \leq \mathcal{F}_{0}+\left\langle\mathcal{H}-\mathcal{H}_{0}\right\rangle_{0} \equiv \mathcal{F}_{\mathrm{MF}} \\
& \mathcal{H}_{0} \equiv \frac{T}{2} \sum_{\mathbf{q}} S^{1}(\mathbf{q}) h_{\mathbf{q}} h_{\mathbf{q}}
\end{aligned}
$$

This is a variational approach in which the propagator, $S(\mathbf{q})$, itself is the variational parameter. When particularizing the mean field free energy, $\mathcal{F}_{\mathrm{MF}}$, to two dimensions, a single, first-order phase transition is found.

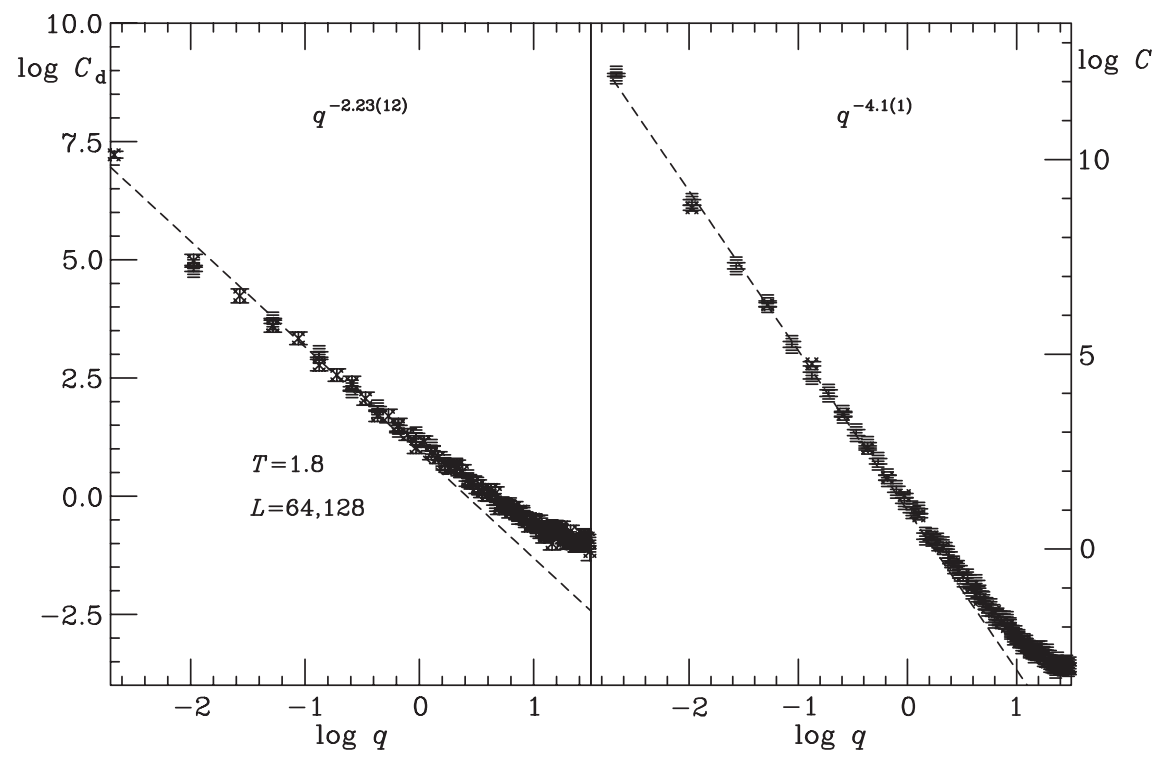

Fig. 1. Propagators. $T 1.8$. 


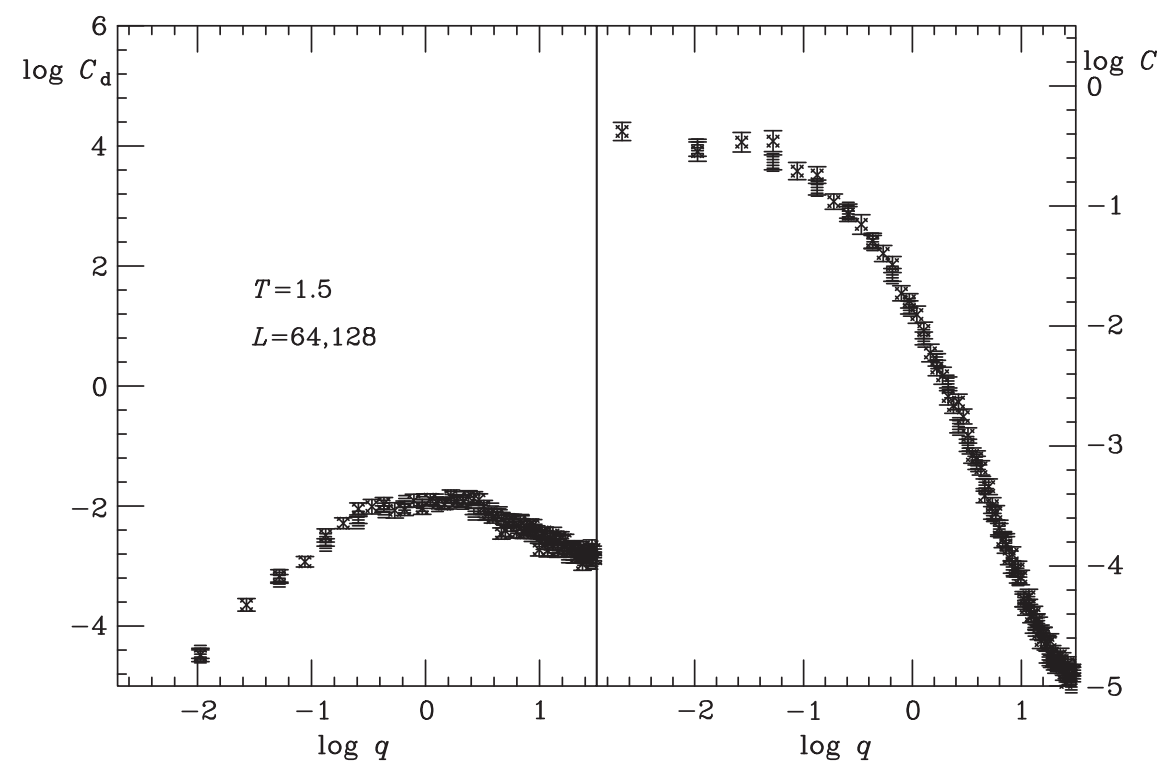

Fig. 2. Propagators. $T$ 1.5.

Renormalization Group Scenario [8]. Renormalization begins with the Langevin equation for the continuum limit [7] of model (3), which is renormalized using the scheme of Nozières-Gallet [10]. In a very sketchy manner, we can summarize this approach as follows: let us define $L_{\mathrm{c}}(l)=\sqrt{ } \kappa(l) / v(l), l=\log b, b$ being the scale factor in $\mathrm{RG}$; then, it is found that, if $L<L_{\mathrm{c}}(l)$, the surface is tensionless, otherwise surface tension is generated. In addition, for large lattices $(l \rightarrow \infty)$ there is always surface tension and, as a consequence, there is a single KT phase transition, but the high temperature phase is as for the Gaussian roughening model [described in the continuum by
Eq. (1) for $\kappa=0]$, rather than as for the bilaplacian model [Eq. (1) for $v=0]$.

Therefore, according to the approaches we have summarized above, there can be one or two phase transitions in the Laplacian roughening model, that can be KT-type or first order, properties of the high temperature phase being unclear. This is a rather unsatisfactory situation that calls for further research. Coming now to the available simulation data, we point out that numerical simulations performed by Bruce [4] seem to confirm the Nelson scenario with $T_{\mathrm{cl}}=2.2$ and $T_{\mathrm{c} 2}=1.64$. However, results obtained by Janke and Kleinert [5] in a modified model would imply a first-order

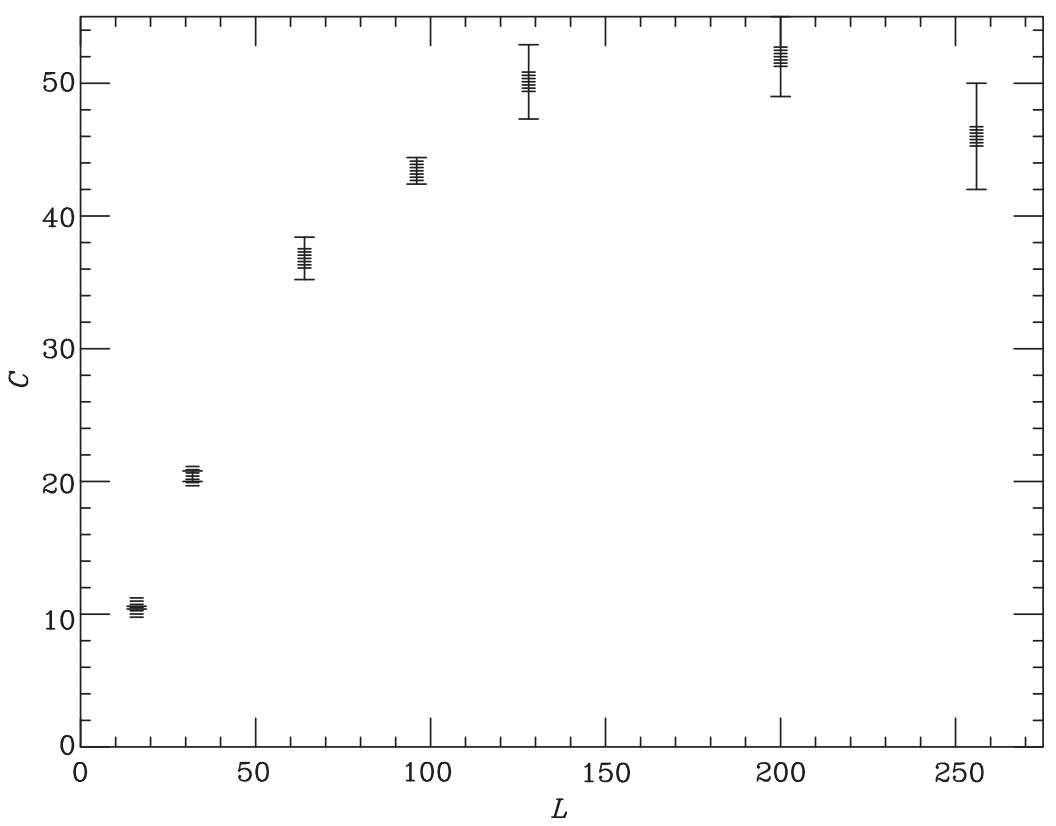

Fig. 3. Peak of the specific heat as a function of $L$. 


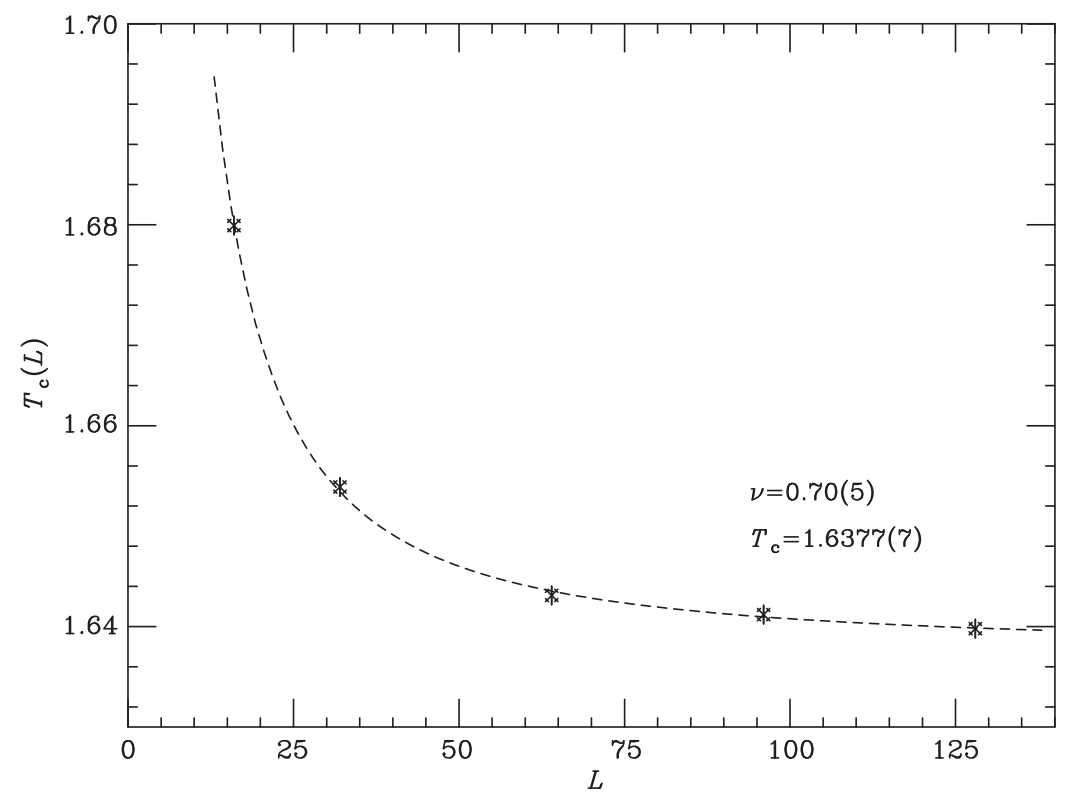

Fig. 4. $T_{\mathrm{c}}(L)$ as a function of $L$. Fit to Eq. (4).

phase transition in the Laplacian roughening model at $T_{\mathrm{c}}=2.454$, in contradiction with Bruce. We thus see that the numerical simulations have not shed any light on the discrepancy so far, hence the need for the additional work we are reporting here.

\section{Numerical simulation results}

In this section, we report on the results of our large-scale numerical simulation program designed to test the different scenarios we have seen in the previous section. To this end, we begin by computing the normal and slope propagators at $T=1.8$. In Fig. 1, it can be seen that both propagators follow the continuum bilaplacian prediction $\left(1 / q^{4}\right.$ and $1 / q^{2}$ behaviors, respectively), hence neither the Bruce scenario nor the Janke and Kleinert scenario can be valid, at least not with the critical temperatures reported by them.

We have repeated the computation in the low temperature region $T=1.5$. Fig. 2 shows a dramatic change of behavior: The slope-slope propagator shows a bell shape and the normal propagator shows a plateau in the low momentum regime, indicating a massive phase (or, in other words, a finite correlation length). We can explain these two behaviors assuming a massive continuum bilaplacian model, which has $1 /\left(q^{4}+m^{4}\right)$ as the normal propagator, where the mass $m$ is the inverse of the correlation length of this phase. This behavior for the normal propagator implies a behavior

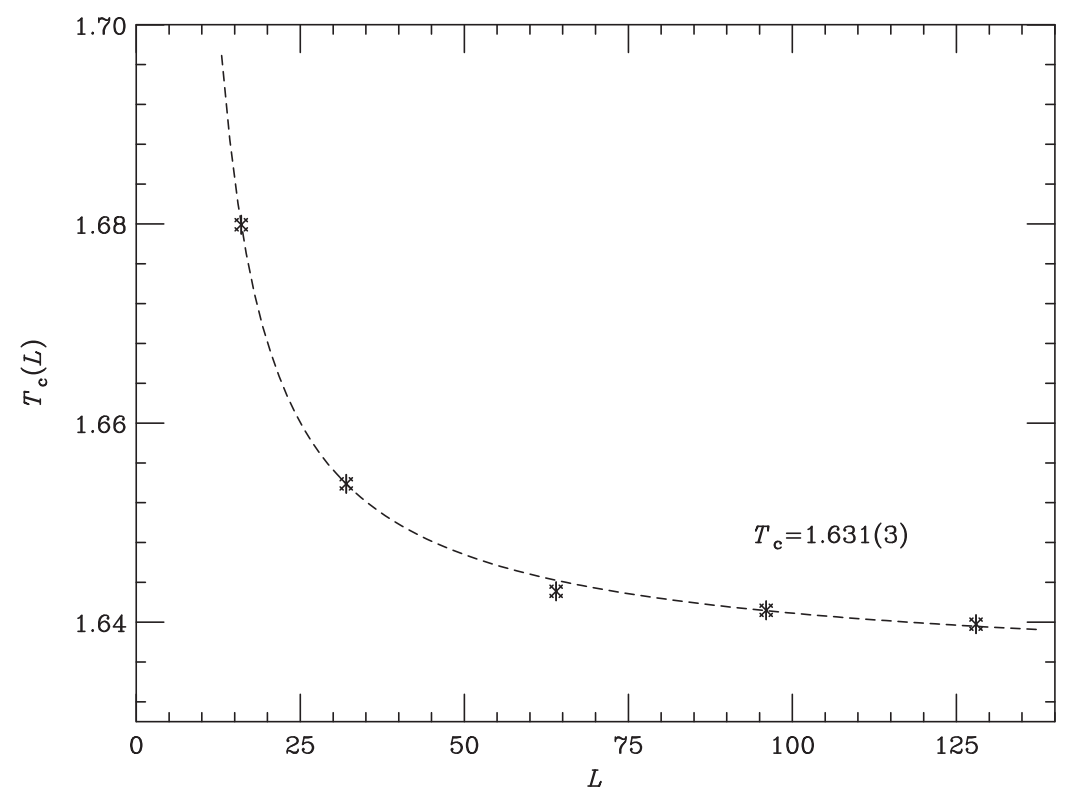

Fig. 5. $T_{\mathrm{c}}(L)$ as a function of $L$. KT scenario: fit to Eq. (4). 
$q^{2} /\left(q^{4}+m^{4}\right)$ for the slope-slope propagator, which indeed presents the bell shape. It is very important to make clear that in Figs. 1 and 2 there are no finite size effects within our numerical accuracy: data for $L=64$ and for $L=128$ are compatible up to statistical errors.

As an additional check, we have studied the behavior of the specific heat. This observable shows a clear maximum as a function of temperature for a given lattice size. We have plotted the value of that maximum as a function of the lattice size in Fig. 3. In the low $L$ region linear behavior is observed, for large lattices a plateau seems to have been achieved. This behavior resembles that of the peak of the specific heat in a KT phase transition, and implies a specific heat critical exponent $\alpha \leq 0$, i.e., there is no divergence of the specific heat. We can do further analysis by monitoring the dependence of the apparent critical temperature, $T_{\mathrm{c}}(L)$ (defined as the temperature in which the specific heat shows the maximum for a given lattice size) on the lattice size. This apparent critical temperature scales, in an ordinary second order phase transition, as [11]

$$
T_{\mathrm{c}}(L)=T_{\mathrm{c}}+a L^{1 / v} .
$$

In Fig. 4 we plot $T_{\mathrm{c}}(L)$ versus $L$ and the fit to Eq. (4). We obtain $v=0.70(7)$. However, this value of $v$, along with our result that $\alpha \leq 0$; implies violation of the hyperscaling relation [11] $\alpha=2-v d$, for $d=2$. Hence, we try a fit motivated by the KT scenario [11]:

$$
T_{\mathrm{c}}(L)=T_{\mathrm{c}}+\frac{a}{(\log L+g)^{2}},
$$

where $a$ and $g$ are constants, and $T_{\mathrm{c}}$ is the bulk critical temperature. As can be seen from Fig. 5, this fit is in very good agreement with the numerical data. Nevertheless, a definitive assessment of the order of the transition requires further research, which will be presented elsewhere [12].

\section{Conclusions}

Having presented our numerical simulation results, we are in the proper position to discuss them in the light of previous work and to draw some conclusions from that comparison. To begin with, the outcome of our simulations makes us confident that the system behaves in a continuum massless bilaplacian way at $T=1.8$, i.e., at that temperature the model exhibits an infinite correlation length. This result contradicts those reported by Bruce [4], for whom the system should be in Nelson's hexatic phase, and by Janke and Kleinert [5], who found the transition at a much higher temperature. Furthermore, we have seen no sign at all of the two-transition scenario proposed by Nelson [3]; indeed, the system undergoes a continuous phase transition near $T=1.64$ (where the system develops a non-diverging specific heat peak) between a high temperature phase, well described by the bilaplacian, and a low temperature one which can be described by a massive (finite correlation length) quartic propagator. However, further studies are needed to exclude the presence of a quadratic term (which would indicate surface tension effects) in this propagator (i.e., $1 / C(q)=$ $\left.a q^{4}+b q^{2}+c\right)$. To be more specific about the possibility of having two phase transitions, on the grounds of our simulations we are confident ${ }^{2}$ that propagators at $T=1.64$ behave as at $T=1.5$, meaning that $T=1.64$ would still be the low temperature phase. Conversely, at $T=1.7$ the propagators are still as at $T=1.8$, i.e., we are in the high temperature phase. Hence, a detailed study of the region near $T \in(1.64$; $1.7)$ is needed in order to discard completely the intermediate hexatic phase proposed by Nelson (work along this line is in progress, and will be reported elsewhere [12]). As for the type of the transition we observe, the choice for the critical exponents $\alpha \leq 0$ and $v \simeq 0.7$ violates hyperscaling in two dimensions, so that the phase transition might be in the KT universality class. The behavior observed in the specific heat would reinforce this conclusion.

To summarize, our numerical results are not compatible with the Nelson scenario (two phase transitions), the variational approach (a single first order phase transition) and with the RG (that differs in the properties of the high temperature phase). We hope that further numerical research will lead to a final conclusion, although we believe that the results we are reporting here should not be far from it. Once the correct scenario is assessed, it will be interesting to try to give an accurate analytical description of it.

\section{Acknowledgments}

Partial financial support from CICyT (Spain) grants BFM2001-0718, BFM2003-08532-C03-02 (JJRL), BFM2003-07749-C05-01 (RC and AS), and BFM200204474-C02 (EM) is acknowledged. E.M. acknowledges a Ramón y Cajal contract by the Spanish MEC.

\section{References}

[1] S. Leibler, Statistical mechanics of fluctuating films and membranes, in: D. Nelson, T. Piran, S. Weinberg (Eds.), Statistical Mechanics of Membranes and Surfaces, Cambridge University Press, 1998.

[2] A. Pimpinelli, J. Villain, Physics of Crystal Growth, World Scientific, Singapore, 1996.

[3] D. Nelson, Laplacian roughening models and two-dimensional melting, Phys. Rev., B 26 (1982) 269

[4] D.A. Bruce, Phase transitions in the Laplacian roughening model, Mater. Sci. Forum 4 (1985) 51.

[5] W. Janke, H. Kleinert, Monte Carlo study of two-step defect melting, Phys. Rev., B 41 (1990) 6848.

[6] E. Moro, R. Cuerno, A. Sánchez, Growth dynamics of crystalline tensionless surfaces, Phys. Rev. Lett. 78 (1997) 4982.

\footnotetext{
2 The maximum lateral lattice size we have studied, $L \quad 256$, is at least four times larger than those of previous simulations in the literature.
} 
[7] E. Moro, R. Cuerno, Variational mean-field study of a continuum model of crystalline tensionless surfaces, Phys. Rev., E 63 (2001) 036104.

[8] R. Cuerno, E. Moro, Dynamic renormalization group study of a generalized continuum model of crystalline surfaces, Phys. Rev., E 65 (2002) 016110.

[9] N. Gov, S.A. Safran, Pinning of fluid membranes by periodic harmonic potentials, Phys. Rev., E 69 (2004) 011101.
[10] F. Nozières, F. Gallet, The roughening transition of crystal surfaces: I. Static and dynamic renormalization theory, crystal shape and facet growth, J. Phys. (Paris) 48 (1987) 353.

[11] C. Itzykson, J.-M. Drouffe, Statistical Field Theory, Cambridge University Press, 1989.

[12] R. Cuerno, E. Moro, J.J. Ruiz-Lorenzo (in preparation). 